\title{
Denoising the Underwater Images by using Adaptive Filters
}

\author{
${ }^{1}$ Azra Jeelani and ${ }^{2}$ Veena M B \\ ${ }^{1}$ Research Scholar, Department of Electronics \& Communication Engineering, BMS College of \\ Engineering, Bengaluru-560019, Karnataka, India. \\ ${ }^{2}$ Associate professor, Department of Electronics \& Communication Engineering, BMS College of \\ Engineering, Bengaluru-560019, Karnataka, India. \\ azrajeelani@gmail.com, veenamb.ece@bmsce.ac.in
}

\begin{abstract}
The Sound Navigation And Ranging and synthetic aperture radar images are perturbed by the multiplicative noise called speckle noise. The presence of speckle noise leads to incorrect analysis and has to be handled carefully. Images have a strong variation from one pixel to another which reduces the efficiency of the algorithms for detection and classification. In this paper, the most well-known filters are analyzed by using underwater images. It is shown that they are based on a test related to the local coefficient of variation of the observed image, which describes the scene heterogeneity. Linear noise removing models can remove noise but are not able to preserve edges of the images in an efficient manner. On the other hand, Non-linear models can handle edges in a much better way than linear models. It is found that the linear filters and nonlinear filters can remove noise from small area objects and homogeneous areas but not in heterogeneous areas. Adaptive filters are used to remove noise not only from homogeneous area but also from heterogeneous areas. This paper presents an optimum filter by using locally estimated parameter values to provide minimum mean square error in order to smoothen the sonar images. It is also shown that the optimum filter is computationally efficient. The performance of this adaptive filter is compared (qualitatively) with other filters and found that the complexity of the frost filter is reduced.
\end{abstract}

Keywords: Speckle noise, adaptive filter, mean square error, homogeneous areas and heterogeneous areas.

\section{Introduction}

Image restoration is an area that also deals with improving the appearance of an image. However, unlike enhancement, which is subjective, image restoration is objective, in the sense that restoration techniques tend to be based on mathematical or probabilistic models of image degradation. The field of image restoration is concerned with the reconstruction or estimation of the uncorrupted image from a blurred and noisy one. Image restoration is done by restoration filters. Restoration filters are used to reduce the degradations which occur due to sensor noise, blur, camera misfocus, relative object-camera motion and random atmospheric turbulence.

Restoration refers to removal or minimization of known degradations in an image. This includes deblurring of images degraded by the limitations of a sensor or its environment, noise filtering and correction of geometric distortion or non-linearties due to sensors fundamental result in filtering theory. Generally speaking there are two techniques of removing/reducing speckle noise[1], i.e., multi-look process and spatial filtering[12]. Multi-look process is used at the data acquisition stage 
while spatial filtering is used after the data is stored. No matter which method is used to reduce/remove the speckle noise, they should preserve radiometric information, edge information and last but not least, spatial resolution. These are the conditions that any speckle noise reduction technique should meet. Spatial filters are mainly categorized into two general groups ,i.e., nonadaptive and adaptive filters. Non-adaptive filters are those which neglect the local properties of the terrain backscatter or nature of sensor. Non adaptive filters are classified as linear and non linear filters. However, adaptive filters accommodate the change in the local properties of the terrain backscatter or the nature of sensor. Linear filters are averaging(mean)filter and wiener filter[9]. Nonlinear filters are median filter[8][12][2].

\section{The Existing Filtering Methods}

\subsection{Weiner Filter}

Wiener Filter[9]is to filter out noise that has corrupted a signal. It is based on a statistical approach. Typical filters are designed for a desired frequency response. The Wiener filter approaches filtering from a different angle. One is assumed to have knowledge of the spectral properties of the original signal and the noise, and one seeks the LTI filter whose output would come as close to the original signal as possible.

The Wiener filter is:

$$
\mathrm{G}(\mathrm{u}, \mathrm{v})=\frac{H *(u, v) \operatorname{Ps}(u, v)}{[H(u, v)]^{2} \operatorname{Ps}(u, v)+\operatorname{Pn}(u, v)}
$$

Dividing through by $\mathrm{P}_{\mathrm{s}}$ makes its behaviour easier to explain:

$$
\mathrm{G}(\mathrm{u}, \mathrm{v})=\frac{H *(u, v)}{[H(u, v)]^{2}+\frac{P n(u, v)}{P s(u, v)}}
$$

where

$H(u, v)=$ Degradation function and $u, v$ are picture elements(pixels) of an image.

$\mathrm{H}^{*}(\mathrm{u}, \mathrm{v})=$ Complex conjugate of degradation function

$P_{n}(u, v)=$ Power Spectral Density of Noise

$P_{s}(u, v)=$ Power Spectral Density of un-degraded image. The term $P_{n} / P_{s}$ can be interpreted as the reciprocal of the signal to noise ratio.

\subsection{Averaging Filter:}

Averaging Filter: Average (or mean) filtering is a method of 'smoothing' images by reducing the amount of intensity variation between neighbouring pixels. The average filter works by moving through the image pixel by pixel, replacing each value with the average value of neighbouring pixels, including itself. We can use linear filtering to remove certain types of noise. The Mean Filter is a linear filter which uses a mask over each pixel in the signal. Each of the components of the pixels which fall under the mask are averaged together to form a single pixel. This filter is also called as average filter. The Mean Filter is poor in edge preserving.

$$
\hat{f}(\mathrm{x}, \mathrm{y})=\frac{1}{M N} \sum_{(s, t) \in S} g(s, t)
$$




\subsection{Median Filter:}

Median filtering is a nonlinear method used to remove noise from images. It is widely used as it is very effective at removing noise while preserving edges. It is particularly effective at removing 'salt and pepper' type noise. The median filter works by moving through the image pixel by pixel, replacing each value with the median value of neighbouring pixels. The pattern of neighbours is called the "window", which slides, pixel by pixel over the entire image. The median is calculated by first sorting all the pixel values from the window into numerical order, and then replacing the pixel being considered with the middle (median) pixel value.

$$
\begin{aligned}
\hat{f}(\mathrm{x}, \mathrm{y})= & \operatorname{median}\{\mathrm{g}(\mathrm{s}, \mathrm{t})\} \\
& (\mathrm{s}, \mathrm{t}) \in \mathrm{S}_{\mathrm{xy}}
\end{aligned}
$$

\subsection{Adaptive filters:}

An adaptive filter is a system with a linear filter that has a transfer function controlled by variable parameters and a means to adjust those parameters according to an optimization algorithm. An adaptive filter iteratively adjusts its parameters during scanning the image to match the image generating mechanism. Adaptive Filters reduces speckle noise in homogeneous areas, preserves the texture and high frequency information in heterogeneous areas[12].Adaptive filters take into account local image statistics while carrying out the filtration process, and hence they are able to remove the noise while preserving the texture. The different types of adaptive filters are lee Filter[3], Kuan Filter[5][6], Frost Filter[4][13], MAP(Maximum A Posteriori)[10],Modified Lee[13], Modified Frost filters[13] and SRAD(Speckle reducing anisotropic diffusion)[14] Filter. The Lee and Kuan filters have the same formation, although the signal model assumptions and the derivatives are different. The Frost filter also strikes a balance between averaging and the all pass filter. In this case, the balance is achieved by forming an exponentially shaped filter kernel that can vary from a basic average filter to an identity filter on a point wise adaptive basis. Compared to all the adaptive filters, frost filter gives best results. It reduces the noise not only in homogeneous areas but also in heterogeneous areas. This results in computational complexity. The computational complexity is reduced.

\subsection{Performance parameters for comparative analysis}

\subsubsection{Mean Square Error}

Mean Square Error is the ratio of the square of difference between the input and output image to the size of the image.

$$
\mathrm{MSE}=\frac{1}{M N} \sum_{1}^{M} \sum_{1}^{N}(f 1(i, j)-f 2(i, j))^{2}
$$

Here $\mathrm{f} 1, \mathrm{f} 2$ are the input and output images respectively. $\mathrm{M}$ and $\mathrm{N}$ are the sizes of the images. The MSE should be less, which means that the pixel intensity of the input and output image should be as close as possible.

\subsubsection{Peak Signal to Noise ratio (PSNR)}

Peak Signal to Noise ratio (PSNR) is the logarithmic value of the ratio of size of the image and the mean square error of the image.

$$
\mathrm{PSNR}=10 \log \frac{2552}{M S E}
$$

PSNR should be as large as possible which means that the content of signal in the output is large and the noise is less. 


\section{Adaptive Filters}

Contrary to the standard filters, the adaptive filters take local image information into consideration while carrying out the filtration process. Adaptive filters can reduce speckle noise in homogeneous areas while preserving texture and high frequency information in heterogeneous areas. The design of each speckle suppression filter is based on different criteria and parameters and the performance of each speckle filter may vary from one sonar to another; therefore, no generic de-speckling algorithm exists [09]. Consequently, selection of the right de-speckling filter for a specific environment and sensor is a difficult task. Frost [13] filter produces the best results, but as with all the speckle filters, it was not intended for real time processing and is the most computationally complex. The proposed algorithm reduces computational complexity which in turn reduces the elapsed time. The Frost filter reduces speckle noise and preserves important image features at the edges with an exponentially damped circularly symmetric filter that uses local statistics within individual filter windows.

A brief description of adaptive filters is given below

\subsection{Lee Filter}

Lee approximated the multiplicative noise model with a linear one, followed by applying a Minimum Mean Square Error (MMSE) criterion [13]. Then an adaptive filter was formulated as

$$
R(x, y)=l b(x, y)(1-W(x, y))+W(x, y) I(x, y)
$$

where $\mathrm{I}$ is the acquired image, $\mathrm{Ib}$ is the average of an acquired image in a filter window, and $\mathrm{W}$ is a weighting function given by

$$
\mathrm{W}(\mathrm{x}, \mathrm{y})=1-\left\{\mathrm{C}_{\mathrm{u}}{ }^{2} / \mathrm{C}_{\mathrm{I}}^{2}(\mathrm{x}, \mathrm{y})\right\}
$$

$C_{u}=\sigma_{u} / u b, C_{1}(x, y)=\sigma_{l}(x, y) / l b(x, y)$, are the variation coefficients of the noise and the acquired image, respectively. The $\sigma_{u}$ and ubar is noise standard deviation and mean respectively. $\sigma_{l}(x, y)$ and $\operatorname{lbar}(x, y)$ is the image standard deviation and mean in the area around $(x, y)$.

\subsection{Frost Filter}

This filter is different from Lee filter. It estimates the observed scene by convolution of the acquired image with the impulse response of the imaging system. The imaging system impulse response is obtained by using the minimal mean square error (MMSE) criterion. Then the resulting filtering process can be defined by a convolution of the acquired image with the filter kernel.

Weighting Function

$$
W(x, y)=K_{1} e^{-K} d C_{1}(x, y) * \operatorname{sgrt}(x 2+y 2)
$$

$\mathrm{C}_{\text {si-standard speckle index (measure of speckle reduction) }}$

$\mathrm{C}_{1}$-varied standard speckle index

$K_{d}$--damping Factor-defines the extent of smoothing-default value 1

$$
\mathrm{C}_{\mathrm{si}}=\frac{\mu}{\sigma}
$$

Filtering Formula:

$$
\mathrm{R}=\mathrm{I}^{*} \mathrm{~W}
$$




\section{A New Optimized Filter}

\subsection{Modified Frost filter}

The frost filter increases the computational complexity. This is due to more number of computations. From equation 2.2.1

- Computational complexity is due to following operations involved in calculating in weighting function

- 2 squares, square root, 3 multipliers, exponent, multiplier

- Square root replaced by $x+y$

- Exponent function replaced by using taylors series

$$
e^{x}=1+\frac{x}{1 !}+\frac{x^{2}}{2 !}+\frac{x^{3}}{3 !}+\cdots, \quad-\infty<x<\infty
$$

Hence it takes more elapsed time for execution. This elapsed time is reduced by modifying the window function as given below.

$$
W(x, y)=K_{1}\left[1-K_{d} C_{1}(x, y)(X+Y)\right]
$$

\subsection{This reduces the number of computations and hence computational complexity is reduced.}

\section{Experiments and Analysis}

The experiments are performed on following underwater images:

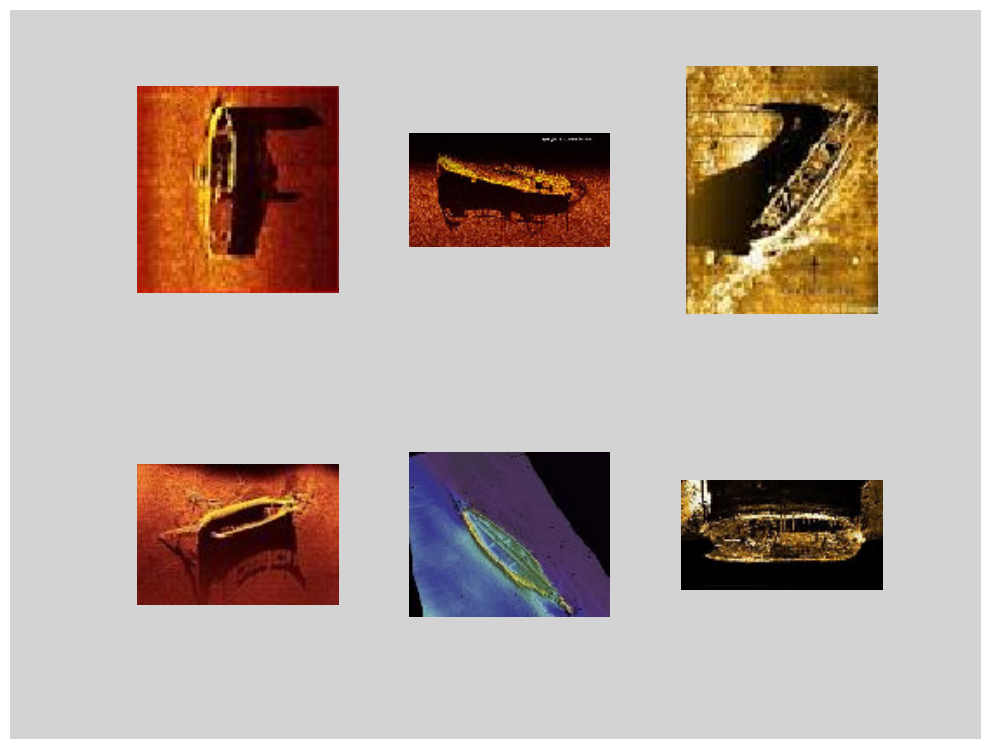

Figure 1: Underwater images 
Table 2. Comparison of filters with respect to mse and psnr.

\begin{tabular}{|l|c|c|}
\hline \multicolumn{1}{|c|}{ Filter } & MSE & PSNR \\
\hline Linear filter-mean filter or averaging filter & 7502 & 9.1 \\
\hline Non Linear filter-median filter & 201 & 25 \\
\hline Non Linear Filter-Wiener Filter & 128 & 27 \\
\hline Adaptive Filter- Lee Filter & 306.6 & 23.2 \\
\hline Adaptive Filter- Frost Filter & 165.5 & 24.7 \\
\hline
\end{tabular}

From the Table II, wiener filter has the least mean square error. It is observed that the linear filters and nonlinear filters can remove noise from small area objects and homogeneous areas but not in heterogeneous areas. Adaptive filters are used to remove noise not only from homogeneous area but also from heterogeneous.

Table.3 Comparison of Frost filter and proposed filter results.

\begin{tabular}{|c|c|c|c|c|}
\hline Image & $\begin{array}{c}\text { Frost } \\
\text { Filter-MSE }\end{array}$ & $\begin{array}{c}\text { Frost Filter- } \\
\text { Elapsed time }\end{array}$ & $\begin{array}{c}\text { Optimized } \\
\text { Filter: MSE }\end{array}$ & $\begin{array}{c}\text { Optimized Fiter: } \\
\text { Elapsed time }\end{array}$ \\
\hline Image 1 & 165 & 2.35 secs & 165 & 0.205 secs \\
\hline Image 2 & 221 & 109 secs & 221 & 3.99 secs \\
\hline Image 3 & 539 & 2.18 secs & 539 & 0.255 secs \\
\hline Image 4 & 161 & 2.7 secs & 167 & 0.27 secs \\
\hline Image 5 & 35 & 20 secs & 35 & 1.5 secs \\
\hline Image 6 & 241 & 24 secs & 241 & 1.509 secs \\
\hline
\end{tabular}

From the Table III, it is observed that mean square error and power signal to noise ratio for two images using frost filter. The complexity of frost filter is due to more number of computations which is reduced modifying the frost filter. This reduces the number of computations hence the elapsed time for execution is reduced.

Original gray image and Frost Filtered output

- Elapsed time is 0.462370 seconds.

- $\quad$ mse1 $=165.5576$

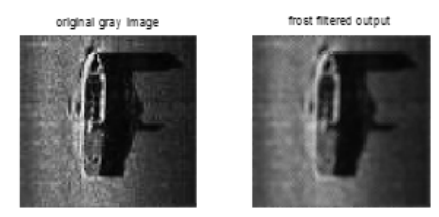

Figure 2: original image and frost filtered image

Original gray image and frost filtered output after reducing the computational complexity 
- $\quad$ Elapsed time is 0.127528 seconds.

$-\quad \operatorname{mse1}=165.5576$
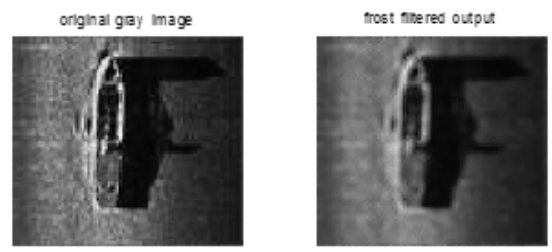

Figure 3: original image and optimum filtered image

\section{Conclusion}

In this work all the classical filters like Lee, Frost, Kuan, GMAP filters, SRAD, linear and nonlinear filters were compared with MSE and PSNR for identifying a best filter. Frost filter was proved to be best but it was found to be computationally complex. Here frost filter is designed with less complexity and reduced elapsed time. The existing filters do not enhance edges-they only inhibit smoothing near edges.

\section{REFERENCES}

[1] J.W.Goodman, "Some fundamental properties of speckle noise", J.opt.Soc.am, vol.66,No.11, 1976, Pg.1145-1150

[2] Otis Lamont Frost, “An Algorithm For Linearly Constrained Adaptive Array Processing”, Proceedings of the IEEE, Vol. 60, No. 8, Aug 1972.

[3] J. S. Lee, "Digital image enhancement and noise filtering by use of local statistics," IEEE Trans. Pattern Anal. Mach. Intell., vol. PAMI-2, no. 2, pp. 165-168, Feb. 1980.

[4] Victor S.Frost, Jsephine Abbott Stiles, K.S.Shanmugan and Julian C.Holtzman, "A Model for Radar Images and Its Application to Adaptive Digital Filtering of Multiplicative Noise", IEEE Trans. Pattern Anal. Mach. Intell., vol.PAMI-4, no. 2, pp. 157-166, Feb. 1982.

[5] D. T. Kuan, A. A. Sawchuk, T. C. Strand, and P. Chavel, "Adaptive noise smoothing filter for images with signal-dependent noise," IEEE Trans. Pattern Anal. Mach. Intell., vol. PAMI-7, no. 2, pp. 165-177, Feb. 1985.

[6] D.Kuan, A.Sawchuk, T.Strand, and P.Chavel, "Adaptive restoration of images with speckle," IEEE Trans. Acoust. Speech Signal Process.,vol. 35, no. 3, pp. 373-383, Mar. 1987.

[7] Armand Lopes, RidhaTouzi and E.Nezry, "Adaptive speckle filters and Scene Heterogeneity", IEEE Transactions on geosciences and remote sensing, Vol.28, No 6,November 1990.

[8] A. Ben Hamza, P. Luque, J. Martinez, and R. Roman, "Removing noise and preserving details with relaxed median filters," J. Math. Imag. Vision, vol. 11,no. 2, pp. 161-177, Oct. 1999.

[9] Er. Simrat, zEr. Anil Sagar, "Empirical Study of Various Speckle Noise Removal Methods", International Journal of Emerging Technologies in Computational and Applied Sciences (IJETCAS), India,2014. 
Azra Jeelani, Veena M B; Denoising the Underwater Images by using Adaptive Filters. Advances in Image and Video Processing, Volume 5 No 2, April (2017); pp: 1-12

[10] Samuel Foucher, Goze Bertin Benie, and Jean-Marc Boucher, "Multiscale MAP Filtering of SAR Images", IEEE Transactions on image processing, Vol. 10, No. 1, January 2001.

[11] Dr.G.Padmavathi, Dr.P.Subashini, Mr.M.Muthu Kumar and Suresh Kumar Thakur, "Performance analysis of Non Linear Filtering Algorithms for underwater images", (IJCSIS) International Journal of Computer Science and Information Security, Vol.6, No. 2, 2009.

[12] Patidar, Pawan, et al. "Image De-noising by Various Filters for Different Noise”, International Journal of Computer Applications 9.4 (2010): 45-50.

[13] Serge Karabchevsky, David Kahana, Ortal Ben-Harush, and Hugo Guterman , "FPGA-Based Adaptive Speckle Suppression Filter for Underwater Imaging Sonar", IEEE JOURNAL OF OCEANIC ENGINEERING, VOL. 36, NO. 4, OCTOBER 2011.

[14] Yongjian Yu and Scott T. Acton, "Speckle Reducing Anisotropic Diffusion", Senior Member,IEEE, IEEE TRANSACTIONS ON IMAGE PROCESSING, VOL. 11, NO. 11, NOVEMBER 2002. 